\title{
Continuous Visualization of Morphological Changes in Endothelial Cells in Response to Cyclic Stretch*
}

\author{
Shunsuke IWAYOSHI ${ }^{* *}$, Katsuko FURUKAWA** and Takashi USHIDA***
}

\begin{abstract}
Endothelial cells are known to change their shapes in alignment with the direction perpendicular to that of cyclic stretch. However, the mechanisms are still not clear. In this study, we developed a new cell-stretching device which allows continuous observation of cyclically stretched/relaxed cells. Using this apparatus, morphological changes of single cells in response to cyclic stretch $(1 \mathrm{~Hz}, 10 \%, 2$ hours) were visualized and analyzed. The data showed that there were two major patterns of morphological changes of ECs, depending on their initial shapes. While ECs with round shapes at the initial state changed their shapes to become more elongated in a direction perpendicular to that of stretch, cells oriented and elongated in the stretch direction at the initial state changed their shapes to first become rounder and then elongated to align perpendicular to the stretch direction at the second step. These results suggest that cell retraction as well as elongation is important in the stretch-induced endothelial morphological changes, and that the elucidation of the mechanisms of the morphological changes requires studies of cell retraction, elongation, and their coordination at the single-cell level.
\end{abstract}

Key Words: Biomechanics, Design, Optical Measurement, Endothelial Cell, Mechanical Stretch, Cell Morphology

\section{Introduction}

Under physiological conditions, blood vessels are exposed to mechanical forces due to the movement of blood, including forces such as shear stress, circumferential cyclic strain, and pulsatile pressure. These mechanical forces regulate blood vessel homeostasis and sometimes cause pathological conditions such as atherosclerosis $^{(1),(2)}$. Vascular endothelial cells (ECs), which compose a confluent monolayer that lines the inner lumen of blood vessels and show spindle-like shapes along the longitudinal axis of the blood vessel, play significant roles as the interface for signal transduction in response to mechanical forces. Numerous reports indicate that mechanical forces affect a variety of endothelial functions such as proliferation and apoptosis in vivo and in vitro ${ }^{(3),(4)}$. One of the

* Received 29th June, 2005 (No. 05-4086)

** Department of Mechanical Engineering, Graduate School of Engineering, University of Tokyo, 7-3-1 Hongo, Bunkyo-ku, Tokyo 113-8656, Japan

*** Center for Disease Biology and Integrative Medicine, Faculty of Medicine, University of Tokyo, 7-3-1 Hongo, Bunkyo-ku, Tokyo 113-0033, Japan.

E-mail: ushida@m.u-tokyo.ac.jp most obvious responses of ECs to mechanical forces is morphological change. For example, in response to applied shear stress, ECs having a round shape and random orientation under the static condition, which is referred to as a cobblestone-like shape, change their shapes to become elongated and oriented in the direction of flow ${ }^{(5)-(7)}$. Cyclic stretch induces cell alignment perpendicular to the stretch direction $^{(8),(9)}$. These morphological changes are believed to be adaptive responses in that they reduce the effective magnitude of mechanical loads to which the ECs are exposed. Many investigations on the mechanisms of shear-flow-induced endothelial alignment have been conducted for decades, and the results suggest that there are particular intracellular signals and molecular components involved in this response, e.g., actin filaments, integrin, and the small GTPases Rho and $\operatorname{Rac}^{(10)-(12)}$. However, less is known about the effects of stretch, because of the difficulties in applying stretch to cells and in the visualization of the cells.

To study the effects of stretch on cells, several types of stretch chambers have been developed and tested for decades ${ }^{(8),(9),(13)}$. Two types of stretch chambers are often used: the uniaxial stretch chamber and the biaxial stretch chamber. These chambers usually have elastic membranes 
such as silicone sheets coated with a cell matrix protein such as fibronectin or laminin, on which the cells are grown. When the membrane is stretched, strain is applied to cells through the basal contact of the cells to the membrane. This way of stretching cells simulates the strain on ECs under the physiological condition, as the EC monolayer in vivo rests on an elastic basement membrane secreted by the ECs. The applied stretch can be a single sustained stretch with different magnitudes and durations or cyclic stretch/relaxation, e.g., sinusoidal, with different amplitudes, durations, and frequencies. In a uniaxial chamber, the rectangular membrane is clamped at two ends while the other two sides are left free. The amount of stretch is set by a mechanical device such as a stepping motor. As the membrane is stretched in one direction, however, it shrinks in the other direction by an amount determined by its Poisson ratio. In a biaxial chamber, a controlled pressure acts to deflect the circularly clamped membrane in a vertical direction, which causes stretching of the membrane in both radial and circumferential directions.

If the membrane is transparent and thin, the device would allow microscopic observation of cells through the membrane. However, it is difficult to observe cells subjected to strain, because the cells being stretched would move out of the visual field in the uniaxial strain chamber and out of focus in the biaxial strain chamber. The observation of cells under cyclic stretch is extremely difficult, particularly when the cells are constantly moving and shifting out of the visual field or focal plane. These difficulties in the visualization of stretched cells have been a critical limitation in stretch stress studies.

In this study, we developed and fabricated a new cellstretching device which allows continuous visualization of the same cells cyclically stretched/relaxed. By using this apparatus, time-lapse observation and analysis of morphological changes of individual ECs under cyclic stretch were achieved. The data shows there were different patterns of endothelial morphological changes depending on their initial cell shapes, indicating that cell retraction as well as elongation play important roles in the morphological changes.

\section{Materials and Methods}

\subsection{Design of cell-stretching device}

A new cell-stretching device has been designed and fabricated in the our laboratory (Fig. 1) for the application of stretch to cells that meets the following requirements

(1) Application of uniaxial strain to living cells.

(2) Microscopic visualization of cells being stretched.

(3) Variation of magnitude of stretch: 5\%, 10\% and $15 \%$.

(4) Application of cyclic sinusoidal uniaxial strain to

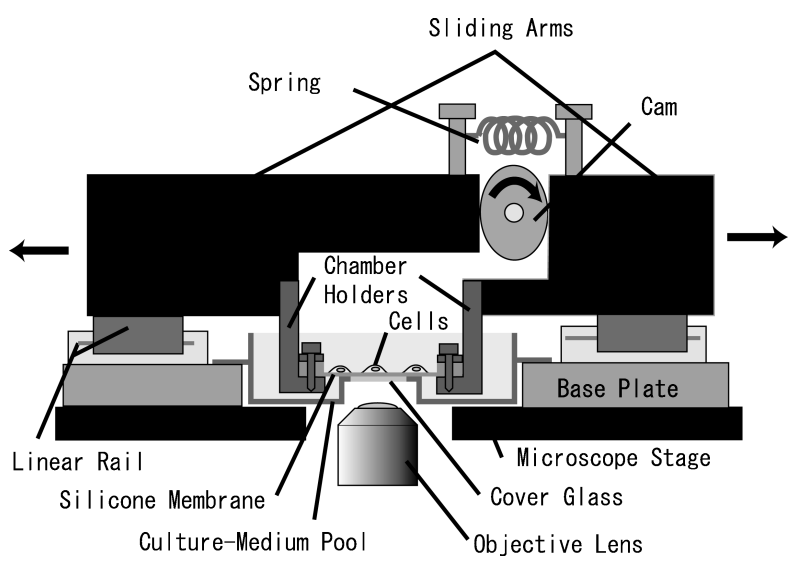

(a)

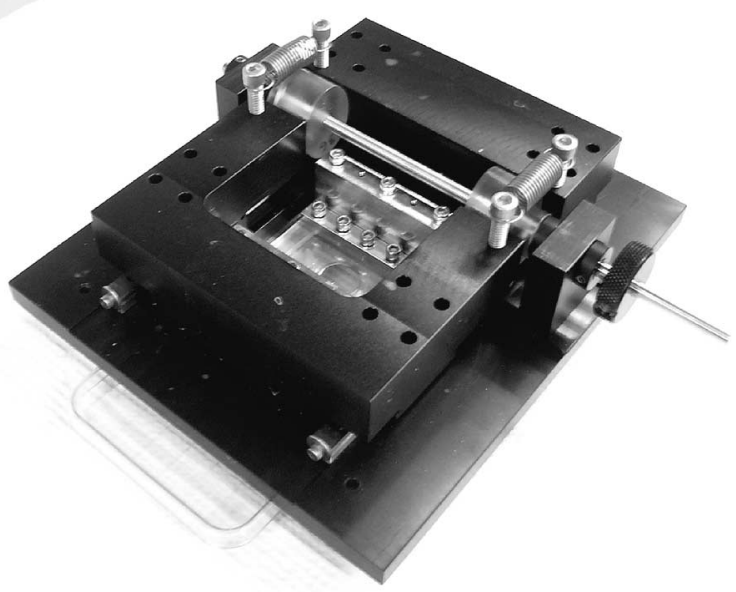

(b)

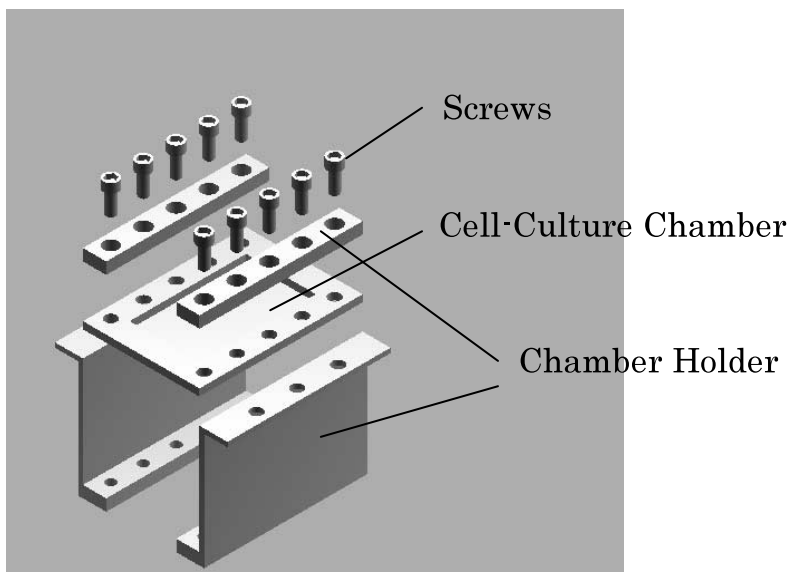

(c)

Fig. 1 Schematic (a) and photograph (b) of cell-stretching device. (c) Schematic of cell-culture chamber and chamber holders

living cells.

These requirements were satisfied through the following design approaches.

(1) The device consists of two main parts: a strain generator and an elastic cell-culture chamber. The strain 
generator includes the chamber holder, cams, sliding arms, linear rails (Nippon Thompson Co.), base plate, and culture-medium pool. The sliding arms and base plate were made of aluminium, cams were made of plastic, and chamber holders and the culture-medium pool were made of stainless steel. The elastic cell-culture chamber was made by gluing thin transparent silicone membrane (125 $\mu \mathrm{m}$ in thickness) and thick sidewalls ( $3 \mathrm{~mm}$ in thickness) using a silicone adhesive (Shinetsu Silicone). Both the silicone membrane and sidewall were purchased from Dow Corning Silicones. The cells were grown on the bottom membrane of the chamber. The culture area was $30 \mathrm{~mm}$ in length along the axis of the stretch direction and $50 \mathrm{~mm}$ in length along the axis perpendicular to the stretch direction, and the culture area was coated with a cell matrix protein such as fibronectin or laminin before cell seeding. The width of the sidewall was $10 \mathrm{~mm}$. Both ends of the elastic chamber had five holes on each sidewall, and the sidewalls were clamped with the chamber holders by tightening the screws through the holes on the sidewalls (Fig. 1 (c)). Since there is no detectable slip between the sidewalls and clamping chamber holders, and the chamber is elastic itself, the application of uniaxial stretch to the cell-culture chamber was expected to cause the cells on the bottom membrane to undergo the same stretch. The free sidewalls of the chamber worked to reduce compression of the chamber along the axis perpendicular to the stretch direction, due to Poisson's effect during stretching.

(2) To observe the cells under the microscope, a thin transparent silicone membrane was used as the bottom of the chamber. This allows the observation of the cells through the membrane with an inverted microscope. The membrane is equally stretched from both ends by rotating the oval cams, and set back to the resting position by the tension of the springs. The center of the membrane remains stationary during the stretch motion.

(3) By changing the cam size, different magnitudes of strain can be applied. In this study, three different cam sizes were used. The major axes of the cams are $31.5 \mathrm{~mm}$, $33 \mathrm{~mm}$, and $34.5 \mathrm{~mm}$, and the minor axis is fixed at $30 \mathrm{~mm}$. Since the membrane is $30 \mathrm{~mm}$ in length, the stretch corresponding to these cam sizes are $5 \%, 10 \%$, and $15 \%$, respectively.

(4) To simulate cyclic strain on ECs in vivo, continuous cam rotation generated by a synchronous motor (SH7PF10N, Japan Servo) enabled cyclic sinusoidal stretching of the cells. The culture-medium pool set beneath the cell-culture chamber reduced the effects of medium evaporation for a long-term culture. The area of the culture-medium pool is about $50 \mathrm{~cm}^{2}$ and the height is about $1.5 \mathrm{~cm}$, so the chamber can contain about $50 \mathrm{cc}$ of culture medium with the cell-culture chamber and chamber holders immersed in the medium. The culture-medium pool had an observation window made of cover glass glued to a hole of the pool, so the use of the culturemedium pool did not prevent observation with an inverted microscope. In addition, the height of the observation window was designed to be at almost the same height as the silicone membrane when the culture-medium pool was fixed to the base plate. Therefore, the observation window diminished vertical movement of the membrane during stretch, which would have been caused by bending of the membrane by its own weight.

\subsection{Strain verification}

To verify that the expected amounts of uniaxial strain were applied to ECs, actual strains of the EC layer at different cam sizes were determined by homogeneous finite strain analysis ${ }^{(14),(15)}$. Briefly, Lagrangian strain, with components of the strain tensor being an exact description of the two-dimensional shape change of the membrane tensor, was calculated from changes in segment lengths between triangularly arranged marker points in microscope images. Vacuoles in ECs located 100-300 $\mu \mathrm{m}$ apart were used as the marker points. Since the twodimensional strain tensor is symmetric, the tensor consists of three independent components, $E_{11}, E_{22}$, and $E_{12}$, and can be solved by using the following equation with the three segment lengths between the three marker points arranged in a triangle.

$$
d s^{2}-d s_{0}^{2}=2 E_{i j} d X_{i} d X_{j} \quad(i, j=1,2)
$$

The coordinate system $X_{1}, X_{2}$, to which this equation refers, is a rectangular Cartesian coordinate system in which axis $X_{1}$ corresponds to the direction of stretch loaded to the membrane, and axis $X_{2}$ is perpendicular to axis $X_{1} . d s_{0}$ is the segment length between markers in the undeformed state, $d s$ is the length in the deformed state, and $d X_{1}$ and $d X_{2}$ are the two-dimensional components of the segment in the undeformed reference state. The two normal strains, $E_{11}$ and $E_{22}$, represent the extension of the membrane in the direction of stretch loading and its perpendicular direction, respectively, while the shear strain, $E_{12}$, measures any change in angle between the stretch axis and perpendicular axis. Therefore, for an ideal uniaxial strain, $E_{22}=E_{12}=0$. Nominal strains (length change divided by initial length), $\varepsilon_{1}$ and $\varepsilon_{2}$, and angle change between the two line elements, $\Delta \theta$, can be calculated from the Lagrangian strains as follows:

$$
\begin{aligned}
& \varepsilon_{1}=\sqrt{2 E_{11}+1}-1 \\
& \varepsilon_{2}=\sqrt{2 E_{22}+1}-1 \\
& \sin (\Delta \theta)=\frac{2 E_{12}}{\sqrt{2 E_{11}+1} \sqrt{2 E_{22}+1}} .
\end{aligned}
$$

\subsection{Cell culture}

Bovine aortic endothelial cells (BAECs) and human umbilical vein endothelial cells (HUVECs) were used for strain calibration and morphological change experiments, respectively. BAECs isolated from bovine aorta were 
maintained up to passage 10 in Dulbecco's modified Eagle's medium (DMEM, Gibco BRL) supplemented with $10 \%$ fetal bovine serum (FBS), $2 \mathrm{mM} \mathrm{L}$-glutamine, and 1 $\mathrm{mM}$ each of penicillin-streptomycin and sodium pyruvate in a humidified $5 \% \mathrm{CO}_{2}-95 \%$ air incubator at $37^{\circ} \mathrm{C}$. HUVECs purchased from Sanko Junyaku were maintained in EBM-2 (Sanko Junyaku) supplemented with 2\% FBS and other supplements, as per the manufacturer's instructions in a humidified $5 \% \mathrm{CO}_{2}-95 \%$ air incubator at $37^{\circ} \mathrm{C}$. HUVECs between passages 4 and 10 were used for experiments. The elastic silicone chambers were sterilized by autoclaving and filled with fibronectin $\left(4 \mu \mathrm{g} / \mathrm{cm}^{2}\right.$, Sigma) for BAECs or laminin $\left(5 \mu \mathrm{g} / \mathrm{cm}^{2}\right.$, Asahi Techno Glass Corp.) for HUVECs in water. After evaporation of the water, the chamber was washed with PBS, and the ECs $\left(50000 \mathrm{cells} / \mathrm{cm}^{2}\right)$ were seeded on the membrane of the chamber and grown to confluency, which usually took two to three days.

\subsection{Morphological analysis of ECs under cyclic stretch}

Cyclic stretch $(10 \%, 1 \mathrm{~Hz})$ was applied to $\mathrm{HU}-$ VECs grown on laminin-coated silicone membranes, using the developed cell-stretching device. Before starting cyclic stretch, the culture medium was replaced with HEPES buffered solution (5.5 mM HEPES, $130 \mathrm{mM}$ $\mathrm{NaCl}, 1.8 \mathrm{mM} \mathrm{CaCl}_{2}, 5.4 \mathrm{mM} \mathrm{KCl}, 0.8 \mathrm{mM} \mathrm{MgCl}_{2}$, $5.5 \mathrm{mM}$ Glucose, $\mathrm{pH}$ 7.4) supplemented with $10 \%$ FBS. The medium was maintained at $34^{\circ} \mathrm{C}$ with a heat plate (IMT2-HP, Olympus) on the microscope stage in room air during the experiments.

Phase-contrast images of ECs were observed with an inverted microscope (IX50, Olympus) and an objective lens $(\times 4)$. Time-lapse movies acquired with a CCD camera (SSC-DC45, SONY) through a photoeyepiece lens $(\times 5)$ were recorded using a computer with a video capture device (USTV2004, Canopus) at a video rate (30 frames/s). Frame images of deforming cells under cyclic stretch and frame images of ECs in the relaxed state at 0 , $15,30,45,60,75,90,105$, and 120 min were acquired from the recorded movies using software (Area61 video browser, Area61.NET). The resolution of the acquired images was $271 \times 203$ pixels where one pixel corresponds to about $4 \mu \mathrm{m}$. To quantify the cell shape, cell edges were traced manually with Photoshop (Adobe), followed by the measurement of the cell area, perimeter length, and orientation angle from the traced images, using Scion Image (Scion Corp.). Measured angles were distributed between 0 and 90 , in which 0 degree corresponds to the direction of stretch. To analyze cell elongation, shape index (S.I.) was calculated from the cell area and perimeter length. S.I. was defined as

$$
\text { S.I. }=4 \pi \frac{\text { Area }}{\text { Length }^{2}} .
$$

The value of the S.I. is between 0 and 1: $0 \leq$ S.I. $\leq 1$. S.I. equals unity for a circular cell and approaches zero for a highly elongated cell.

\subsection{Statistical analysis}

Comparisons of the results between two groups were performed using the paired t-test. P-values of $<0.05$ were considered statistically significant. Each experiment was performed independently a minimum of three times.

\section{Results}

\subsection{Visualization of cells being stretched}

BAECs were cultured on fibronectin-coated silicone membranes, and visualization of the BAEC layer being stretched was tested with the new developed device. Although visualization was realized at $4 \mathrm{x}$ magnification at $10 \%$ stretch, higher magnification and larger strain caused vertical movement of membranes such that they became out of focus. By using the observation window of the culture-medium pool as a membrane supporter, we found that the vertical movement of the membrane was reduced and the visualization of stretching cells, which was realized up to $10 \%$ stretch and 20x magnification, was improved. However, over $15 \%$ stretch or $40 \mathrm{x}$ magnification, the cells shifted out of focus due to vertical movement of the membrane and visualization became unclear.

\subsection{Strain calibration}

Because the cell-stretching device allows the observation of cells being stretched, exact strain of the BAEC layer could be calculated from microscope images of the EC layer with and without stretch. To verify the exact strain, the Lagrangian strain tensor of the EC layer was calculated from the phase-contrast images of stretched and unstretched ECs grown on silicone membranes (Fig. 2). The Lagrangian strain tensors of the EC layer at $5 \%$ stretch were $E_{11}=0.058 \pm 0.004($ mean $\pm \mathrm{SD}, n=3), E_{12}=0.000 \pm$ 0.003 , and $E_{22}=-0.005 \pm 0.003$. The Lagrangian strain tensors of the EC layer at $10 \%$ stretch were $E_{11}=0.104 \pm$ $0.004, E_{12}=0.005 \pm 0.003$, and $E_{22}=-0.012 \pm 0.003$. The Lagrangian strain tensors of the EC layer at $15 \%$ stretch were $E_{11}=0.148 \pm 0.004, E_{12}=0.002 \pm 0.006$, and $E_{22}=-0.008 \pm 0.012$.

Since the shear component $E_{12}$ was not significantly different from zero, we assumed $E_{12}$ to be negligible, and calculated nominal strain $\varepsilon$ (length change divided by initial length) by solving equations (2) and (3). Nominal strain $\varepsilon_{1}$ was $5.6 \pm 0.4 \%, 10.0 \pm 0.3 \%$, and $13.8 \pm 0.3 \%$ at $5 \%, 10 \%$, and $15 \%$ stretch, respectively, and nominal strain $\varepsilon_{2}$ was $-0.5 \pm 0.7 \%,-1.2 \pm 0.3 \%$, and $-0.8 \pm 1.2 \%$, respectively.

\subsection{Visualization of cyclically stretched/relaxed HUVECs}

To visualize dynamic cell deformation under cyclic stretch $(1 \mathrm{~Hz}, 10 \%)$, movies of cyclically stretched/relaxed HUVECs were recorded. Figure 3 shows frame images during 1 cycle, which corresponds to 30 frames. Arrows 

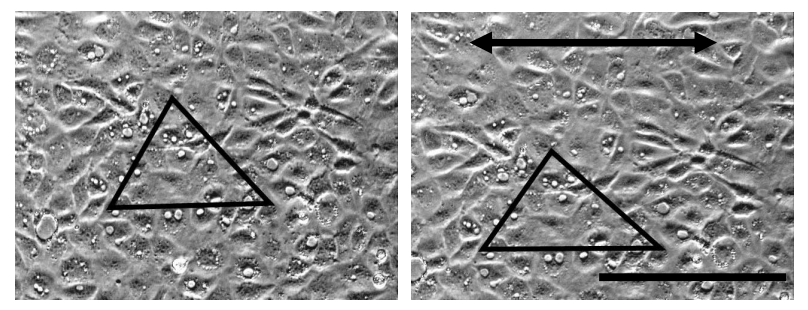

(a)

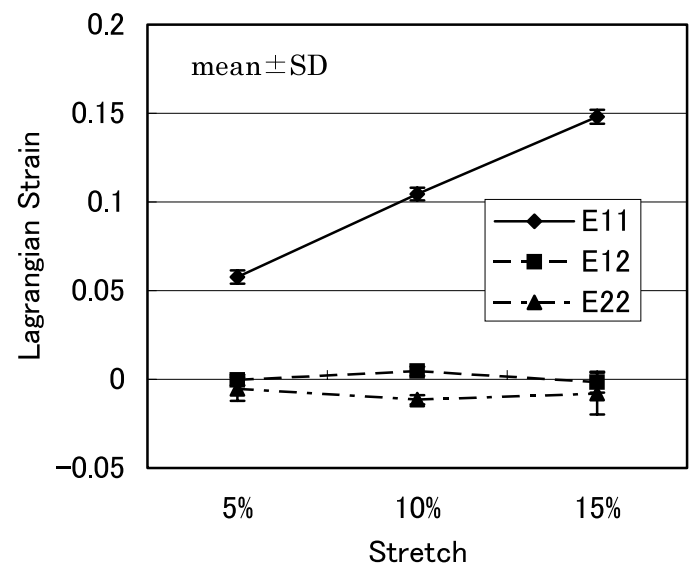

(b)

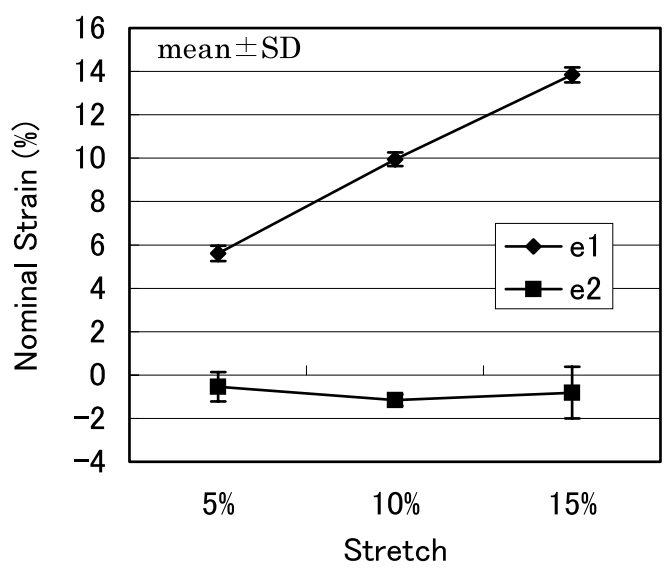

(c)

Fig. 2 (a) Microscope images (20x) of BAEC layer in undeformed state (left) and deformed state (right). Direction of stretch is indicated by black arrow. The resolution of the images is $640 \times 480$ pixels. Bar length $=200 \mu \mathrm{m}$ (b) Lagrangian strain on EC layer vs. stretch at different cam sizes. (c) Nominal strain on EC layer vs. stretch at different cam sizes. $n=3$ different chambers

in Fig. 3 indicate the same cell in the different images. Although most cells remained within the microscopic visual field, there was slight horizontal movement. In these images, there is no detectable blur due to vertical or horizontal movement of the membrane.

\subsection{Morphological changes of HUVECs}

Cyclic stretch $(1 \mathrm{~Hz}, 10 \%)$ was applied to HUVECs for two hours, and microscope images were recorded as movies. Figure 4 shows frame images of ECs in the relaxed state at $0,15,30,45,60,75,90,105$, and 120
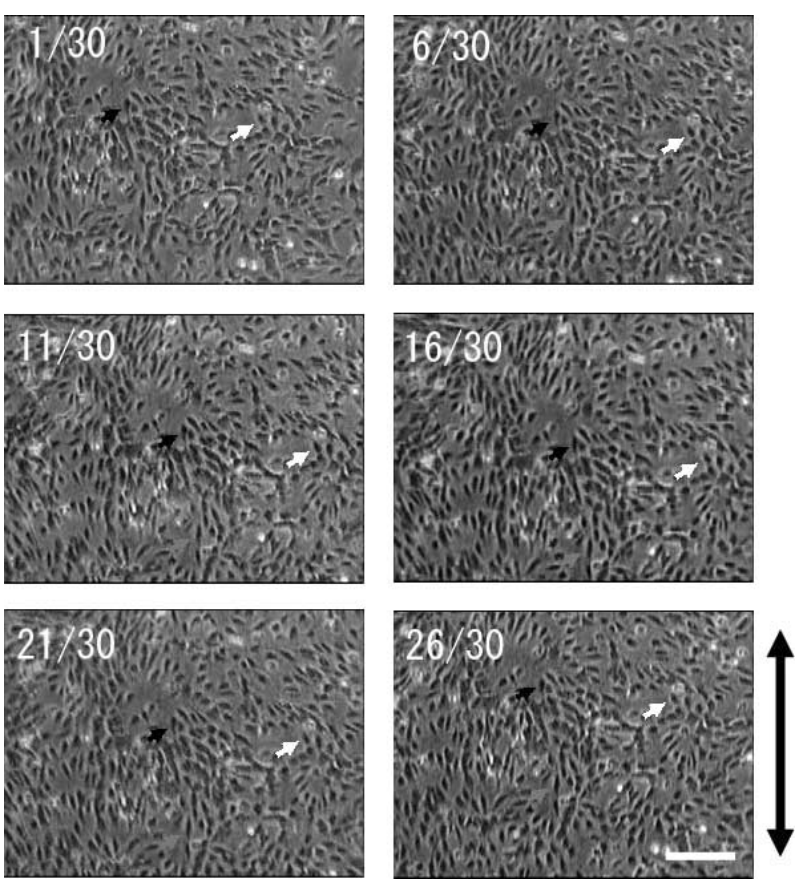

Fig. 3 Frame images of stretching ECs in one cycle. One cycle corresponds to 30 frames, and images every five frame are shown in the figure. $1 / 30$ and 16/30 correspond to ECs in fully relaxed state and fully stretched state, respectively. Identified cells are indicated by arrowheads. Direction of stretch is indicated by black arrow. Bar length $=200 \mu \mathrm{m}$
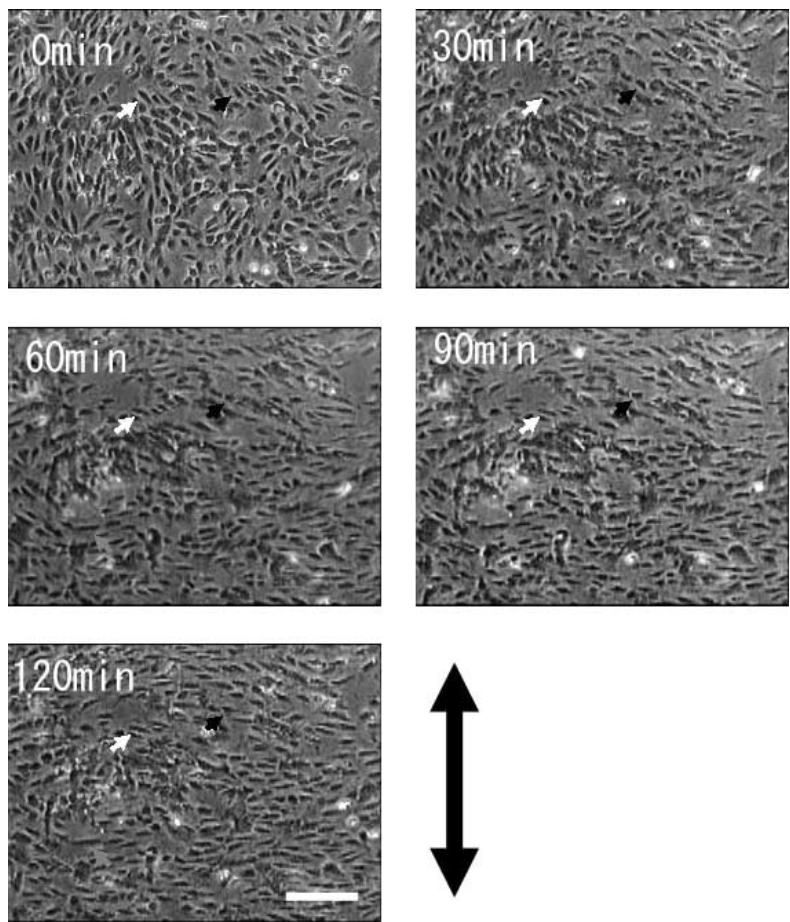

Fig. 4 Morphological changes of ECs in response to cyclic stretch. Images at $0,30,60,90$, and $120 \mathrm{~min}$ are shown. Identified cells are indicated by arrowheads. Direction of stretch is indicated by black arrow. Bar length $=200 \mu \mathrm{m}$ 


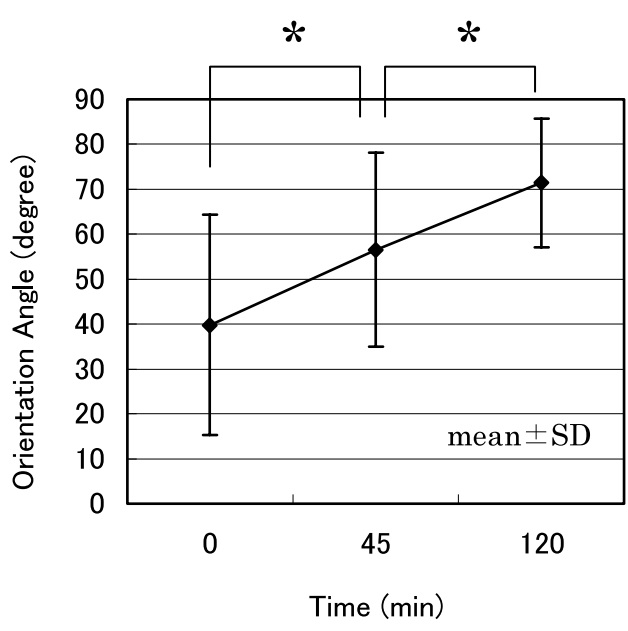

(a)

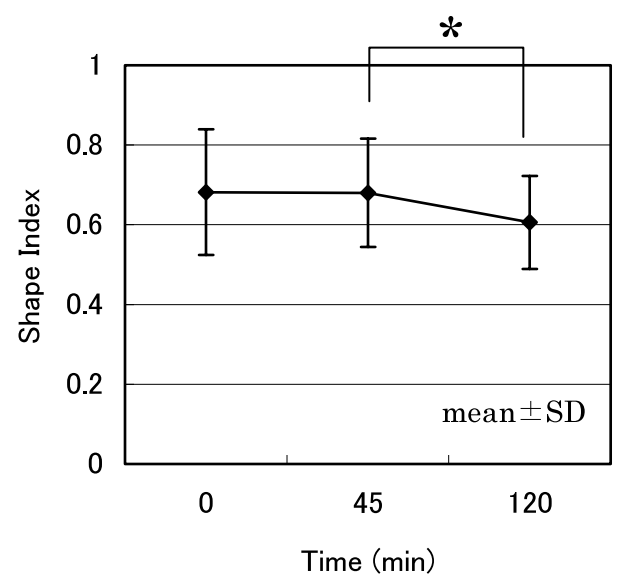

(b)

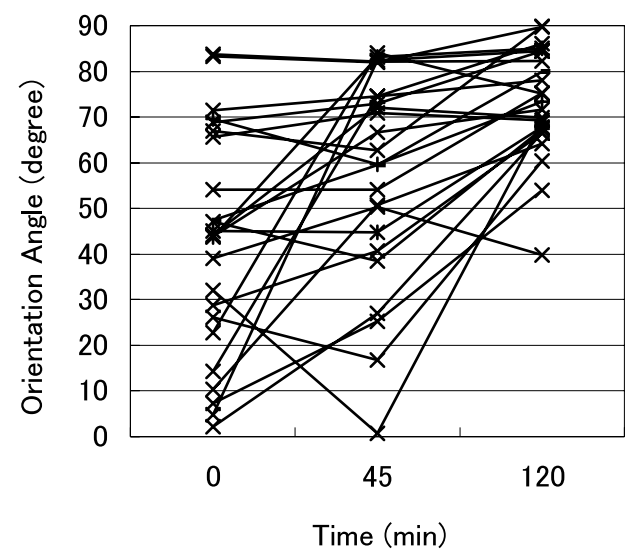

(c)

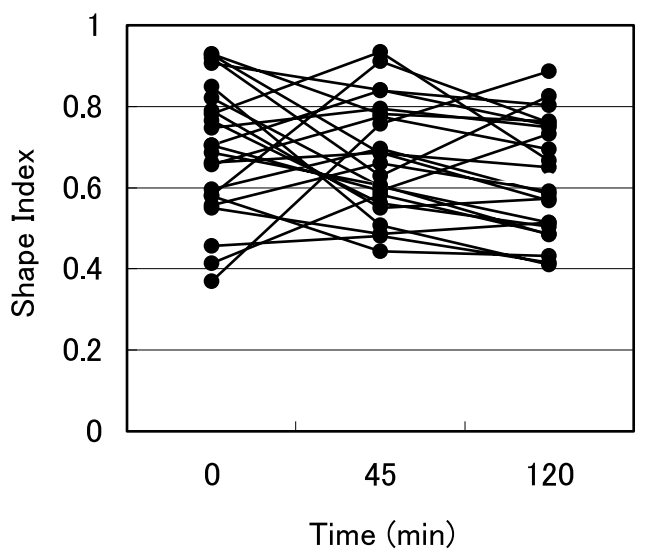

(d)

Fig. 5 Mean angle \pm SD (a) and mean shape index \pm SD (b) of ECs at 0 min, 45 min and 120 $\min (n=93$ cells). Angle changes (c) and shape index changes (d) of single ECs. 0 degree corresponds to the stretch direction. ${ }^{*} P<0.05$ by t-test

min, acquired from a movie. As shown in Fig. 4, ECs, which were randomly oriented before stretching, gradually aligned and elongated in a direction perpendicular to that of stretch during the two hours of observation. To analyze the cell morphological changes, mean cell angle \pm SD and mean S.I. $\pm \mathrm{SD}$ at $0 \mathrm{~min}, 45 \mathrm{~min}$, and $120 \mathrm{~min}(n=93$ cells, four independent experiments) were measured from the microscope images (Fig. 5 (a) and (b)). The mean angle, which was 45 degrees at 0 min, increased significantly during the two hours of study and approached 90 degrees. In addition, the standard deviation of the angles decreased at the same time. These results indicate that ECs which were oriented randomly at $0 \mathrm{~min}$ gradually aligned in a direction perpendicular to that of stretch as a function of time. S.I. did not change significantly between $0 \mathrm{~min}$ and $45 \mathrm{~min}$, but decreased significantly from $45 \mathrm{~min}$ to 120 min. This result indicates that ECs at $120 \mathrm{~min}$ became more elongated than ECs at $0 \mathrm{~min}$ and $45 \mathrm{~min}$.

\subsection{Morphological analysis of single ECs}

Because the new cell-stretching device can apply cyclic stretch without loss of visualization, cells at different time points were identified, and morphological changes of single cells could be analyzed (Fig. 5 (c) and (d)). As shown in Fig. 5 (c), most cells gradually became oriented perpendicular to the direction of stretch from 0 $\min$ to $120 \mathrm{~min}$. Figure 5 (d) shows that there were different patterns of S.I. changes with time for different cells, though the overall result can be described by the results in Fig. 5 (b).

To explain the difference in the time courses of changes in S.I., we hypothesized that some cells with a relatively high $S$.I. at 0 min changed their shapes to become more elongated at $45 \mathrm{~min}$, and that other cells which had a relatively low S.I. and were first oriented in the direction of stretch at $0 \mathrm{~min}$ become rounder at $45 \mathrm{~min}$. To test this hypothesis, all cells were divided into three groups according to their initial shapes: Group I which consists of cells whose initial S.I. was over 0.7 (43 cells), Group II which consists of cells whose initial S.I. was under 0.7 and whose angles were under 45 degree ( 34 cells), and Group 


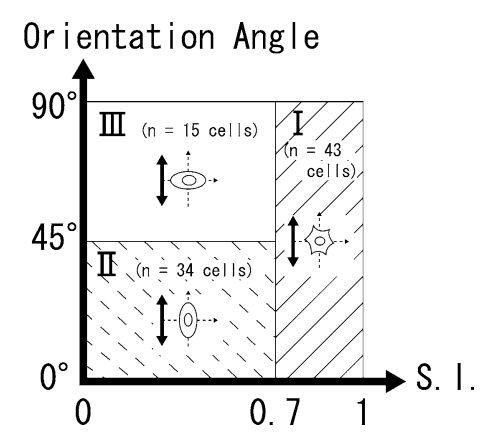

(a)

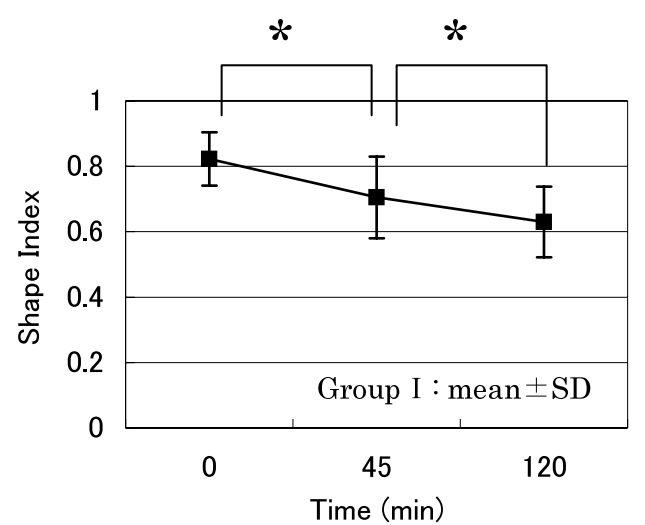

(b)

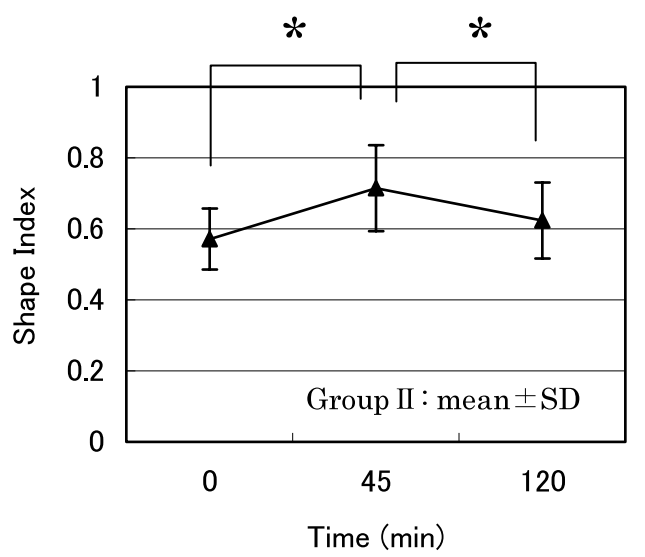

(c)

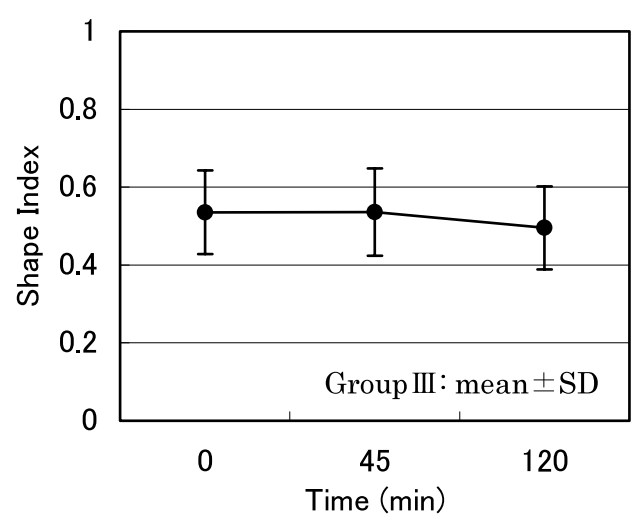

(d)

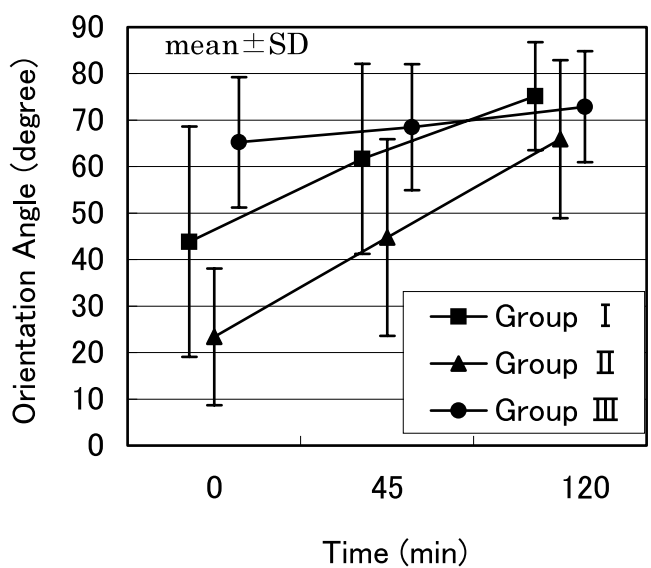

(e)

Fig. 6 (a) Grouping of ECs according to their initial orientation angles and S.I. (b) Shape index changes of ECs in Group I. (c) Shape index changes of ECs in Group II. (d) Shape index changes of ECs in Group III. (e) Angle changes of ECs in Group I, Group II, and Group III. ${ }^{*} P<0.05$ by t-test

III which consists of cells whose initial S.I. was under 0.7 and angles were over 45 degree ( 15 cells) (Fig. 6 (a)). Then S.I. changes of the ECs in each group were analyzed. S.I. of the ECs in Group I significantly decreased from 0 min to $45 \mathrm{~min}$ and from $45 \mathrm{~min}$ to $120 \mathrm{~min}$ (Fig. 6 (b)). On the other hand, ECs in Group II showed significantly increased S.I. from $0 \mathrm{~min}$ to $45 \mathrm{~min}$, which then decreased from $45 \mathrm{~min}$ to $120 \mathrm{~min}$ (Fig. $6(\mathrm{c}))$. At $120 \mathrm{~min}$, S.I. for the two groups became the same. Group III did not show any significant changes in S.I. during the two hours of study (Fig. 6(d)). Although there was a difference in S.I. changes between the three groups, orientation angles showed monotonic increases in all groups (Fig. $6(\mathrm{e})$ ).

To explain how cells in Group I and Group II actually changed their shapes, time-course changes with shorter intervals of cell angle and S.I. of two cells are shown in 

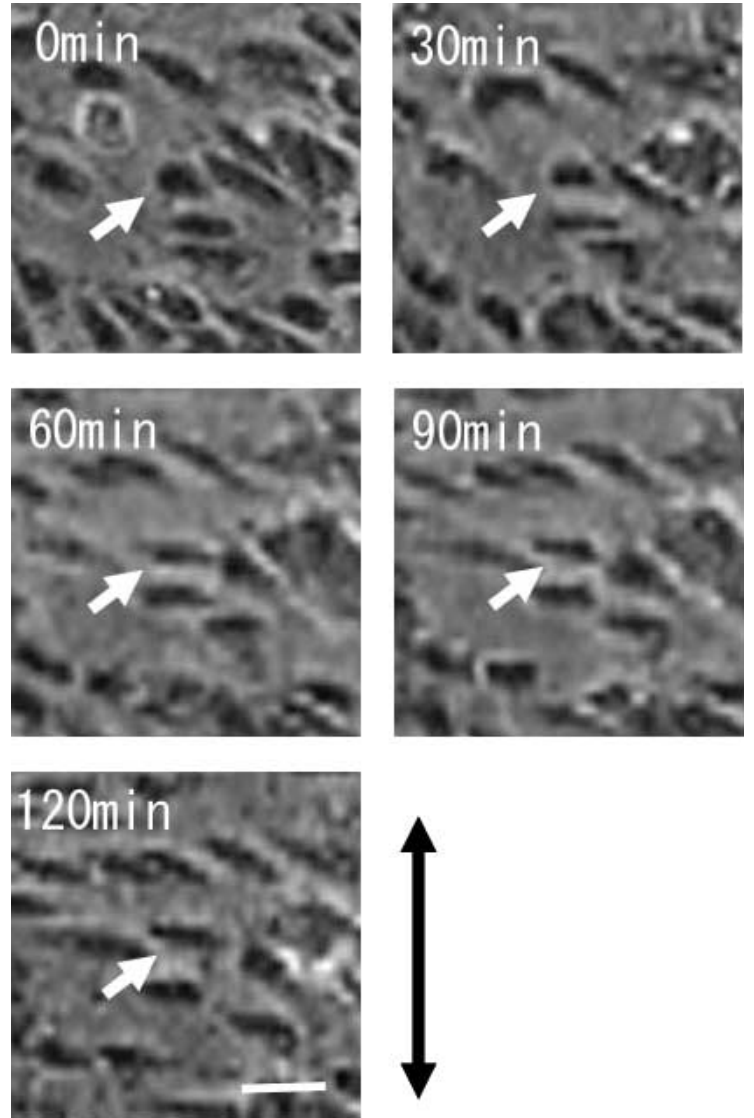

(a)

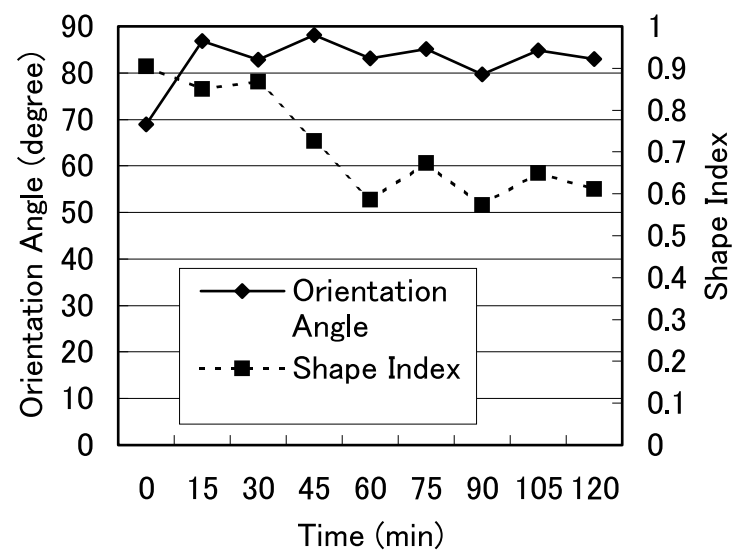

(b)

Fig. 7 Morphological changes of a cell which had round shape at $0 \mathrm{~min}$ (white arrow). Time-course changes of angle (solid line) and shape index (dashed line) of the cell. Direction of stretch is indicated by black arrow. Bar length $=50 \mu \mathrm{m}$

Figs. 7 and 8. As shown in Figs. 7 (a) and (b), a cell with S.I. over 0.9 , round shape and angle close to a direction perpendicular to that of stretch at $0 \mathrm{~min}$ became more perpendicularly oriented relative to the stretch direction right after the onset of cyclic stretch, and gradually elongated over the two hours of study. On the other hand, in Fig. 8 (a) and (b), a cell which was aligned in the stretch
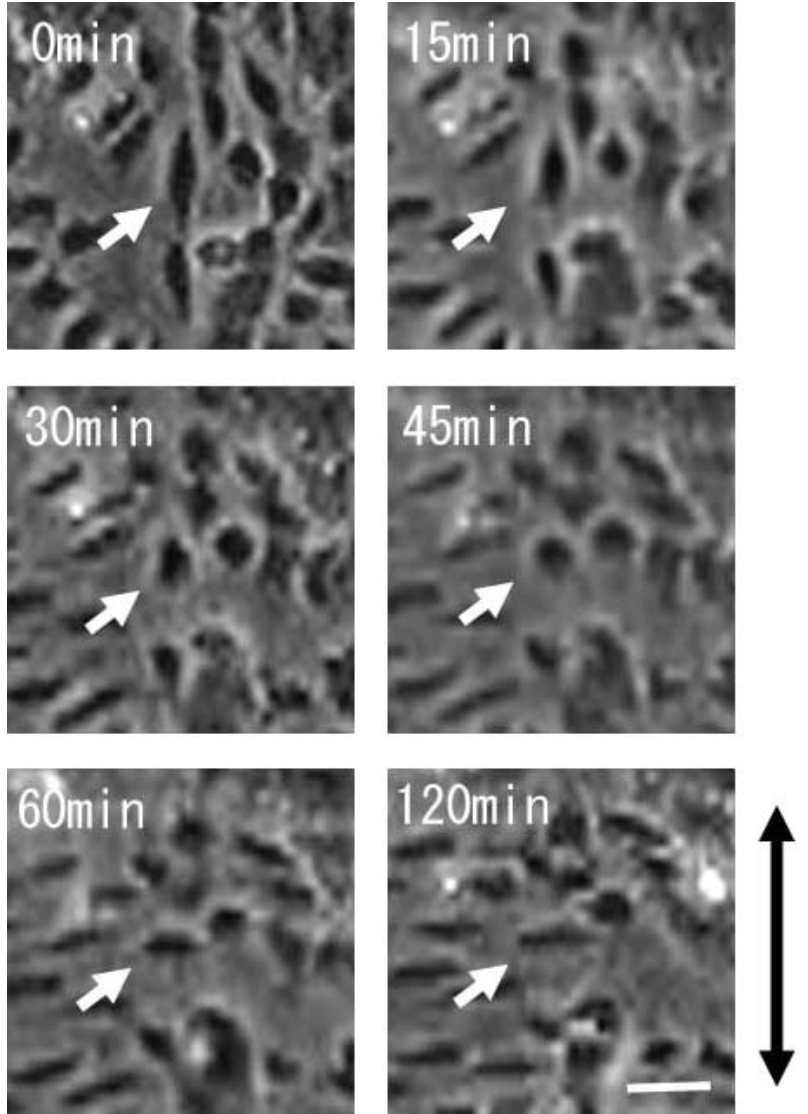

(a)

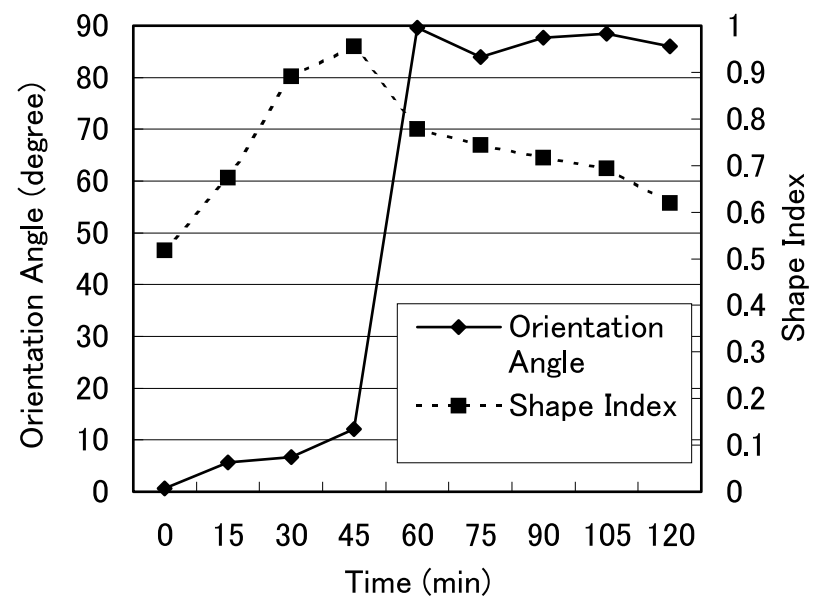

(b)

Fig. 8 Morphological changes of a cell aligned in the stretch direction at 0 min (white arrow). Time-course changes of angle (solid line) and shape index (dashed line) of the cell. Direction of stretch is indicated by arrow. Bar length $=50 \mu \mathrm{m}$

direction before cyclic stretch gradually retracted and became rounder at $45 \mathrm{~min}$, and then elongated from $45 \mathrm{~min}$ to $120 \mathrm{~min}$ to become oriented perpendicular to the stretch direction.

\section{Discussion}

A new device for applying uniaxial stretch to cells 
grown on silicone membrane under microscopic observation was developed. Since ECs, in vivo, grow on elastic basal lamina and are exposed to circumferential stretch, the method of applying stretch using the new device is similar to that in vivo. Because the device is designed to enable the visualization of cells being stretched, exact strains on the EC layer were easily calculated from microscope images. Since $E_{12}$ was not significantly different from zero, shear stress is negligible on the device. Nominal strain $\varepsilon_{1}$ showed good agreement with expected strain, particularly at $10 \%$ stretch. $\varepsilon_{2}$ was negative in value, indicating that compression occurs along the perpendicular axis with the application of stretch. Although the silicone membrane had sidewalls to restrict the movement in the direction perpendicular to that of stretch loading, there was still $1.2 \%$ compression with the application of $10 \%$ stretch. Whether this amount of compression can affect cell functions remains unclear. In addition, strains on the membrane at different positions were almost uniform and showed good agreement with strain on the EC layer up to $10 \%$ stretch (data not shown), which was verified by the calculation of Lagrangian strain from microscope images (4x) of the ink-marked membrane, where the ink, instead of cells, was used as markers.

Our device enabled not only visualization of cells with and without stretch, but also could be used to dynamically deform cells under cyclic stretch. Therefore, time-lapse movies of ECs under cyclic stretch could be recorded. Since the ECs in the movies recorded with the new cell-stretching device remained in the screen throughout the experiments, the movies allowed the tracing of single cells as they dynamically changed their shapes during cyclic stretch with higher specificity than conventional cell-stretching devices. This ability of cell identification in the frame images acquired from the movies enabled quantitative analysis of the shape changes of the single cells. The S.I. which we introduced for the quantitative analysis of cell shape might include some errors. One may be caused by the definition of the S.I., which can describe cell shape best when the cell is elliptical. For example, stellate shapes or asymmetrical shapes of cells could cause error to some extent. Another cause is the relatively low resolution of the acquired images. The resolution of the acquired images in this study was about $4 \mu \mathrm{m} / \mathrm{pixel}$, and the resolution of the $4 \mathrm{x}$ objective lens used in this study was about $2 \mu \mathrm{m}$. Considering that the size of ECs is about $50 \mu \mathrm{m}$, this degree of resolution was assumed to be sufficient for their morphological analysis. In addition, this low resolution could enable us to approximate the real cell shapes as ellipsoidal shapes.

As previously reported by several groups ${ }^{(8),(9),(16)}$, we observed endothelial alignment and elongation perpendicular to the direction of stretch after two-hour cyclic uniaxial stretch. However, time-course analysis of the single- cell morphology provided more information which would have been neglected had the ECs been analyzed as a mass. While angles of most cells gradually aligned perpendicular to the direction of stretch, which is the same information obtained by global analysis, S.I. of single ECs showed different patterns of change, depending on their initial shapes. S.I. of ECs in Group I, that is, ECs which had round shapes at the initial state, decreased from $0 \mathrm{~min}$ to $45 \mathrm{~min}$ and from $45 \mathrm{~min}$ to $120 \mathrm{~min}$. On the other hand, S.I. of ECs in Group II, that is, ECs which oriented and elongated in the stretch direction at the initial state, showed an increase from $0 \mathrm{~min}$ to $45 \mathrm{~min}$ and a decrease from $45 \mathrm{~min}$ to $120 \mathrm{~min}$. The results of the shape changes of ECs in Group II suggested that ECs did not align their shapes by simple rotation, because the S.I. should not have increased if the cell alignment was by simple rotation. Single-cell observation provided clues for the elucidation of the mechanisms of this morphological change. A cell which was oriented and elongated in the stretch direction showed gradual retraction until it became round, and then started elongation perpendicular to the stretch direction. This result indicates that there are two distinct processes in cyclic-stretch-induced endothelial morphological changes, namely, retraction and elongation. Although mass analysis of shape changes of ECs in Group II showed an increase and decrease in S.I. and gradual alignment of cell orientation, this might be results of the occurrence of cell retraction and elongation at the same time.

Since the disruption of actin filaments inhibits cyclicstretch-induced endothelial alignment ${ }^{(12)}$ and actin stress fibers align perpendicular to the stretch direction, actin filaments and stress fibers are believed to be important in endothelial alignment in response to stretch. However, the roles of the actin filaments and stress fibers are unclear. Cell contraction is induced by myosin coupled actin stress fibers in ECs. Wang et al. showed that the inhibition of the contractility of actin stress fiber with a myosin activity inhibitor, 2,3 butanedione monoxime (BMD), also prevented cyclic-stretch-induced endothelial morphological changes ${ }^{(17)}$. These previous reports and our findings suggest that cell orientation in response to cyclic stretch is induced by cell retraction caused by stress fiber contraction.

What signal would induce stress fiber contraction and cell rounding. The small GTPase Rho is a possible signal. Rho family GTPases, Cdc42, Rac, and Rho, play roles in cytoskeletal arrangement and cell morphology. The activations of $\mathrm{Cdc} 42$, Rac, and Rho induce filopodia, lamellipodia, and stress fiber formation, respectively ${ }^{(18)}$. In flow studies, many researchers suggested that flow-induced endothelial alignment depends on Rac and Rho activities. In stretch studies, Yano et al. also demonstrated that the small GTPase Rho is also involved in the endothelial morphological changes in response to cyclic stretch ${ }^{(19)}$. How- 
ever, the roles of Rho signaling in this morphological change are unclear. In this study, we show that cell retraction occurs in the early stage in cyclic-stretch-induced endothelial morphological changes. Since Rho activation induces cell contraction by myosin light chain phosphorylation ${ }^{(20)}$ as well as stress fiber formation and is activated in ECs by cyclic stretch for $15 \mathrm{~min}^{(21)}$, the cell retraction observed in our experiments might have been a result of Rho activation.

For further intensive studies of stretch effects on ECs, a helpful experimental technique would be fluorescent microscopy, which allows various kinds of living-cell experiments at the single-cell level, e.g., calcium ion concentration measurement with Fura-2 and molecular distribution analysis with green fluorescent protein (GFP) ${ }^{(22)}$. Moreover, various fluorescent probes for signal transduction measurement by fluorescence resonance energy transfer (FRET) ${ }^{(23),(24)}$ between GFP variants and fluorescent chemicals have been constructed recently. For example, in shear experiments, the activation of the small GTPase Rac1 downstream of ECs was shown by FRET studies ${ }^{(25)}$. However, fluorescent microscopy generally requires visualization with high magnitude and high N.A. because of weak fluorescent intensity. Our cell-stretching device enabled the visualization of ECs under stretch with up to 20x magnification. Although most cells remained in the microscope field of view, there was slight movement in both the direction of stretch loading and the direction perpendicular to that of stretch. This horizontal movement causes difficulties in observation with a high-magnitude objective lens, because of its smaller field of view. There are two possible causes of the horizontal movement of the specimen. The first possible cause is a gap between the center point of the microscope field of view and the center point of the membrane. Gaps along both the axis of the stretch direction and its perpendicular axis could cause the movement. Because the membrane is stretched to both sides to the same extent, ideally, cells on the centerline of the membrane should remain stationary when using our cellstretching device. Gaps in the axis of the stretch direction cause the movement of the specimen along this axis. On the other hand, there is some influence of Poisson's effect with this device, so the gap in the axis perpendicular to the stretch direction causes cell movement along this axis during stretch. The second possible cause is the insufficient accuracy of machining of the oval cams that we used because it is easy to achieve sinusoidal cyclic stretch. Even though the lengths of major and minor axes are sufficiently accurate, which was verified by strain analysis, if the curved line of cams is not precisely ellipsoidal, membrane stretching to both sides in different amounts may occur, resulting in the movement of cells in the stretch direction. To avoid this, the use of a stepping motor instead of cams as a strain generator might be effective. In addi- tion, there is another difficulty for fluorescent microscopy, which is the inapplicability of an oil objective lens to specimens, because of the short working distance of oil objective lenses. For fluorescent microscopy, an oil objective lens is usually used because of its high N.A. Adopting upright microscopy with a water immersion lens would solve this problem.

In summary, we developed and fabricated a new cellstretching device which allows the visualization of stretching cells. By using this apparatus, the observation and analysis of single-cell morphology under cyclic stretch were possible. The data showed that there are different patterns of endothelial morphological changes in response to cyclic stretch, depending on their initial shapes and suggested that retraction as well as elongation plays an important role. The mechanisms by which cyclic stretch induces endothelial morphological changes are still unclear. To elucidate these mechanisms, studies of the mechanisms of cell retraction, elongation, and their coordination at the single-cell level are necessary.

\section{Acknowledgment}

This study is partially conducted under the advice of Drs. Shu Chien and Shunichi Usami at University of California, San Diego. We earnestly thank them for their support and for allowing us to use their facilities to perform the experiments during our visit. One of the authors (S.I) was supported through the 21st Century COE Program, "Mechanical System Innovation," by the Ministry of Education, Culture, Sports, Science and Technology.

\section{References}

( 1 ) Boegehold, M.A., Shear-Dependent Release of Venular Nitric Oxide: Effect on Arteriolar Tone in Rat Striated Muscle, Am. J. Physiol., Vol.271, No.2 (1996), pp. H387-395.

( 2 ) Pedersen, E.M., Oyre, S., Agerbaek, M., Kristensen, I.B., Ringgaard, S., Boesiger, P. and Paaske, W.P., Distribution of Early Atherosclerotic Lesions in the Human Abdominal Aorta Correlates with Wall Shear Stresses Measured in Vivo, Eur. J. Vasc. Endovasc. Surg., Vol.18, No.4 (1999), pp.328-333.

( 3 ) Dardik, A., Chen, L., Frattini, J., Asada, H., Aziz, F., Kudo, F.A. and Sumpio, B.E., Differential Effects of Orbital and Laminar Shear Stress on Endothelial Cells, J. Vasc. Surg., Vol.41, No.5 (2005), pp.869-880.

( 4 ) Tricot, O., Mallat, Z., Heymes, C., Belmin, J., Leseche, G. and Tedgui, A., Relation between Endothelial Cell Apoptosis and Blood Flow Direction in Human Atherosclerotic Plaques, Circulation, Vol.101, No.21 (2000), pp.2450-2453.

( 5 ) Malek, A.M. and Izumo, S., Mechanism of Endothelial Cell Shape Change and Cytoskeletal Remodeling in Response to Fluid Shear Stress, J. Cell Sci., Vol.109 (Pt 4) (1996), pp.713-726.

(6) Noria, S., Xu, F., McCue, S., Jones, M., Gotlieb, A.I. and Langille, B.L., Assembly and Reorientation 
of Stress Fibers Drives Morphological Changes to Endothelial Cells Exposed to Shear Stress, Am. J. Pathol., Vol.164, No.4 (2004), pp.1211-1223.

( 7 ) Galbraith, C.G., Skalak, R. and Chien, S., Shear Stress Induces Spatial Reorganization of the Endothelial Cell Cytoskeleton, Cell Motil. Cytoskeleton, Vol.40, No.4 (1998), pp.317-330.

( 8 ) Shirinsky, V.P., Antonov, A.S., Birukov, K.G., Sobolevsky, A.V., Romanov, Y.A., Kabaeva, N.V., Antonova, G.N. and Smirnov, V.N., MechanoChemical Control of Human Endothelium Orientation and Size, J. Cell Biol., Vol.109, No.1 (1989), pp.331339.

( 9 ) Naruse, K., Yamada, T. and Sokabe, M., Involvement of SA Channels in Orienting Response of Cultured Endothelial Cells to Cyclic Stretch, Am. J. Physiol., Vol.274, No.5 (1998), pp. H1532-1538.

(10) Tzima, E., del Pozo, M.A., Shattil, S.J., Chien, S. and Schwartz, M.A., Activation of Integrins in Endothelial Cells by Fluid Shear Stress Mediates RhoDependent Cytoskeletal Alignment, Embo J., Vol.20, No.17 (2001), pp.4639-4647.

(11) Li, S., Chen, B.P., Azuma, N., Hu, Y.L., Wu, S.Z., Sumpio, B.E., Shyy, J.Y. and Chien, S., Distinct Roles for the Small GTPases Cdc42 and Rho in Endothelial Responses to Shear Stress, J. Clin. Invest., Vol.103, No.8 (1999), pp.1141-1150.

(12) Iba, T. and Sumpio, B.E., Morphological Response of Human Endothelial Cells Subjected to Cyclic Strain in Vitro, Microvasc. Res., Vol.42, No.3 (1991), pp.245254.

(13) Banes, A.J., Gilbert, J., Taylor, D. and Monbureau, O., A New Vacuum-Operated Stress-Providing Instrument That Applies Static or Variable Duration Cyclic Tension or Compression to Cells in Vitro, J. Cell Sci., Vol.75 (1985), pp.35-42.

(14) Villarreal, F.J., Waldman, L.K. and Lew, W.Y., Technique for Measuring Regional Two-Dimensional Finite Strains in Canine Left Ventricle, Circ. Res., Vol.62, No.4 (1988), pp.711-721.

(15) Lee, A.A., Delhaas, T., Waldman, L.K., MacKenna, D.A., Villarreal, F.J. and McCulloch, A.D., An Equibiaxial Strain System for Cultured Cells, Am. J. Physiol., Vol.271, No.4 (1996), pp. C1400-1408.

(16) Kaunas, R., Role of Strain Direction in Endothelial Cell Remodeling and Signal Transduction, Ph.D. Dissertation, University of California, San Diego, La Jolla, CA. (2003).

(17) Wang, J.H., Goldschmidt-Clermont, P. and Yin, F.C.,
Contractility Affects Stress Fiber Remodeling and Reorientation of Endothelial Cells Subjected to Cyclic Mechanical Stretching, Ann. Biomed. Eng., Vol.28, No.10 (2000), pp.1165-1171.

(18) Nobes, C.D. and Hall, A., Rho, Rac, and Cdc42 GTPases Regulate the Assembly of Multimolecular Focal Complexes Associated with Actin Stress Fibers, Lamellipodia, and Filopodia, Cell, Vol.81, No.1 (1995), pp.53-62.

(19) Yano, Y., Saito, Y., Narumiya, S. and Sumpio, B.E., Involvement of rho p21 in Cyclic Strain-Induced Tyrosine Phosphorylation of Focal Adhesion Kinase (pp125FAK), Morphological Changes and Migration of Endothelial Cells, Biochem. Biophys. Res. Commun., Vol.224, No.2 (1996), pp.508-515.

(20) Essler, M., Amano, M., Kruse, H.J., Kaibuchi, K., Weber, P.C. and Aepfelbacher, M., Thrombin Inactivates Myosin Light Chain Phosphatase via Rho and Its Target Rho Kinase in Human Endothelial Cells, J. Biol. Chem., Vol.273, No.34 (1998), pp.21867-21874.

(21) Shikata, Y., Rios, A., Kawkitinarong, K., DePaola, N., Garcia, J.G. and Birukov, K.G., Differential Effects of Shear Stress and Cyclic Stretch on Focal Adhesion Remodeling, Site-Specific FAK Phosphorylation, and Small GTPases in Human Lung Endothelial Cells, Exp. Cell Res., Vol.304, No.1 (2005), pp.40-49.

(22) Westphal, M., Jungbluth, A., Heidecker, M., Muhlbauer, B., Heizer, C., Schwartz, J.M., Marriott, G. and Gerisch, G., Microfilament Dynamics during Cell Movement and Chemotaxis Monitored Using a GFP-Actin Fusion Protein, Curr. Biol., Vol.7, No.3 (1997), pp.176-183.

(23) Miyawaki, A., Llopis, J., Heim, R., McCaffery, J.M., Adams, J.A., Ikura, M. and Tsien, R.Y., Fluorescent Indicators for $\mathrm{Ca}^{2+}$ Based on Green Fluorescent Proteins and Calmodulin, Nature, Vol.388, No.6645 (1997), pp.882-887.

(24) Yoshizaki, H., Ohba, Y., Kurokawa, K., Itoh, R.E., Nakamura, T., Mochizuki, N., Nagashima, K. and Matsuda, M., Activity of Rho-Family GTPases during Cell Division as Visualized with FRET-Based Probes, J. Cell Biol., Vol.162, No.2 (2003), pp.223-232.

(25) Tzima, E., Del Pozo, M.A., Kiosses, W.B., Mohamed, S.A., Li, S., Chien, S. and Schwartz, M.A., Activation of Rac1 by Shear Stress in Endothelial Cells Mediates both Cytoskeletal Reorganization and Effects on Gene Expression, Embo J., Vol.21, No.24 (2002), pp.67916800. 